Network Working Group

Request for Comments: 2664

FYI : 4

Obsoletes: 1594

Category: Informational
R. Plzak

SAIC

A. Wells

UWisc-Mad

E. Krol

Univ IL

August 1999

\title{
FYI on Questions and Answers \\ Answers to Commonly Asked "New Internet User" Questions
}

Status of this Memo

This memo provides information for the Internet community. It does not specify an Internet standard of any kind. Distribution of this memo is unlimited.

Copyright Notice

Copyright (C) The Internet Society (1999). All Rights Reserved.

Abstract

This memo provides an overview to the new Internet User. The intended audience is the common Internet user of today, thus it attempts to provide a more consumer oriented approach to the Internet rather than going into any depth about a topic. Unlike its predecessors, this edition seeks to answer the general questions that an unsophisticated consumer would ask as opposed to the more pointed questions of a more technically sophisticated Internet user. Those desiring a more in-depth discussion are directed to FYI 7 that deals with intermediate and advanced Q/A topics. A conscious effort has been made to keep this memo brief but at the same time provide the new user with enough information to generally understand the Internet.

1. Acknowledgements

The following people deserve thanks for their help and contributions to this FYI Q/A: Chris Burke (Motorola), John Curran (BBN Planet), Albert Lunde (NWU), and April Marine (Internet Engines, Inc.). Last, but not least, thanks are extended to Patricia Harper and Charlotte Nurge. These ladies from South Riding, Virginia, consumer tested this document. 


\section{Questions About the Internet}

\subsection{What is the Internet?}

People use computers to perform a wide assortment of tasks. A connected group of computers is known as a network. Because people are connected via this network, they can use their computers to exchange ideas and information. Some computers are connected directly to the network while others (primarily those in homes) are connected via a telephone line and a communication device known as a modem. By connecting networks together with specialized computers known as routers, people on one network can engage in activities with people on other networks. This INTER-connected group of NETworks is known as the INTERNET.

\subsection{What Can I do on the Internet?}

There is a large variety of activities that users can do on the Internet. These activities include surfing, searching, sending mail, transfering programs and documents, chatting, and playing games.

\section{SURFING}

Surfing is one of the most popular Internet activities. To surf, a user needs a program known as a web browser. The web browser enables the user to connect to a location that contains information. Many locations contain links to other sites that contain related information. These links are usually identified by underlined text that is of a different color from the rest of the text in an article. By clicking on one of these links the user is then connected to that information. This information may be at the same location or may be at a different location. This new information may, in turn, have links to other information. So just like a footnote or reference in a print publication, links can be used to find related or non-related information.

\section{SEARCHING}

Searching involves using a special program known as a seach engine. There are several of these engines that are located at various search sites. The popular web browsers have location information about these search sites. Searching is similar to using a card catalog in a library. Just as a person would look up a topic in a card catalog and find one or more references to that topic with library location information, a search engine provides the user with a list of sites that may contain relevant information. This list is actually a set of links to these sites so that all the user has to do is click on the link to go to the location. Just as different library card 
catalogs will contain different reference cards, different search engines will provide different reference lists.

E-MAIL

E-mail is another very popular activity. It is very similar to sending letters through the post office or notes and memos around the office. It is used to exchange messages between two or more people. Because email can be misunderstood or abused, users should be familiar with email netiquette. For more information see Netiquette Guidelines [FYI 28, RFC 1855].

Many people also participate in mailing lists. Usually a mailing list is dedicated to a particular topic or interest. Some mailing lists are used to provide information to subscribers, such as product update information for something an individual may have purchased while others are used for discussion. In the latter instance people participate in the discussion by sending email to a "list" address which in turn distributes it to all members of a list. Abuse of mail lists is probably the biggest source of junk email (also known as "spam"). Everyone should take care that they aren't the source of junk mail.

FILE TRANSEER

Programs and documents are transferred in several ways. The most common way this is done between individual users is to attach the program or document to an e-mail message. Programs and documents are usually transferred from sites to users using the save feature of a web browser or the file transfer protocol (FTP). Such transfers enable users to obtain a variety of programs, documents, audio files, and video files.

\section{CHAT}

Chat takes place between one or more persons who are on the Internet. Chatting is very similar to going to a party. Just as people congregate in small groups and discuss things, chatters meet in chat rooms to discuss a topic. Chat rooms are generally sponsored or operated by an organization that has an interest in the topic area. For example, an online news organization would have a chat room for chatters to discuss current events. To chat one person writes a message which can be read, as it is being written, by the others who can respond to it in turn. First time chatters should be aware that just as at a party where some people never say anything, so there may be people in the room who are just listening. Also, just like at a party, some people may portray themselves to be someone different than who they really are. Lastly, remember that chatters come and go 
to chat rooms the same way people move about groups at a party.

GAMES

Some people use the Internet to play games. These games can be role playing games, action/adventure games, or online versions of old standbys like chess. Some games require the user to purchase a copy of the game and install it on their computer, while others are played by going to a game site. Just like other forms of game playing, Internet game playing can be challenging, entertaining, and an enjoyable social experience. Don't be afraid to have fun.

\section{OTHER ACTIVITIES}

Other popular activities include electronic shopping, banking, and investing. Many retailers describe and display pictures of their products on the Internet enabling people to buy on line. Shopping also includes purchasing services such as an airline ticket or ordering groceries. Many banks allow people to transfer funds, check available funds, pay bills and other such activities while on the Internet with an account number and ID. Lastly, many people invest while on the Internet in everything from stocks and bonds to real estate. One word of caution, if you are using a credit card, check to see if there are security features in place to protect your credit card information. Reputable sites should tell you how they are protecting your information. If you are in doubt about how your information will be protected, don't use your credit card at that site.

\subsection{What is an Address?}

Two commonly asked questions these days are "What's your e-mail address?" and "What's the URL?" Generally, the first question is asking where to send information, while the second is asking where to get information. The answer to the first question is usually something like mynamedcompany.com. The answer to the second question is usually something like "http://www.newspaper.com". What do these answers mean?

E-MAIL ADDRESS

As stated previously an e-mail address is something like "myname@company.com", pronounced "MYNAME at COMPANY dot COM". An email address consists of two parts that are divided by an "@" sign.

The portion to the left is like the name line on a letter, it identifies a particular person and usually is composed of the person's name. Typical names look like this: 


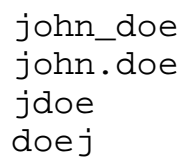

The name is assigned by the system or network adminstrator who is managing the email system and follows rules that have been established by the company providing the e-mail service. Sometimes the name portion of the e-mail address is referred to as a mailbox.

The portion to the right of the "@" sign is the name of the computer system that is providing the e-mail service. This name is usually the name of the company that owns the computer system followed by a "dot" and an abbreviation that represents the "domain" or group of names which the organization falls under. Examples of these "top level" domains are "edu", "com", and country codes such as "fr" for France and "jp" for Japan. When an e-mail is sent the portion of the address to the right of the "@" sign is used to find the destination computer of the email.

URL

A Uniform Resource Locator (URL) is commonly used to identify a computer that provides world wide web service. It usually looks something like "http://www.newspaper.com". This address also consists of two parts. In this case the two parts are separated by the "//". The portion to the left means find the world wide web service that is located at the computer identified to the right of the "//". The portion to the right is the name of the computer that is providing the world wide web service. Its name is composed of parts that are similar to those described for the name of an email computer. Sometimes the portion on the right contains additional information that identifies a particular document at the web site.

For example, http://www.newspaper.com/sports/articlel.html would identify a specific article in the sports section of the newspaper.

\subsection{Are There Any Rules of Behavior on the Internet?}

In general, common sense, courtesy, and decency govern good Internet behavior. There is no single formal rulebook that governs behavior on the Internet. FYI 28 that was mentioned previously is a good guide. Many activities such as game sites, chat rooms, or e-mail lists may have rules of their own. What may be acceptable behavior in one chat room may be totally out of bounds in another. It never hurts to check the water temperature before jumping in the pool. Users should use the same precautions before joining in any online activity. 
E-mail in particular can lead to misunderstandings between people. Users should remember that the reader only has the text to determine what is being said. Other conversation cues such as "tone of voice" and body signals like winking are not present in the text. Because of this, users of the Internet have developed cues to put in the text. Text techniques such as capitalization and symbols known as emoticons (also called "smilies") are used.

$$
\text { A typical smiley looks like this :-) }
$$

Additionally, acronyms have evolved over time (for example IMHO - In My Humble Opinion). More information about this can be found by searching. Use keywords like "netiquette" and "emoticon" with your search engine to find more information.

Users should also be aware that their particular programs such as word processors or e-mail might produce documents and messages that are not readable by everyone. Very often, a reader must have the same program in which a document was written in order to read it. So, before sending an attached document, it is a good idea to make sure that the intended receiver of your document has the capability to read it. If in doubt, send a text (ascii) version of the document.

\subsection{How Does the Internet Work?}

Each of the activities mentioned in the section describing what one can do on the Internet requires that computers exchange information. Computers take turns sending and receiving information. When a computer is sending information, it is known as the "source"; when it is receiving information, it is known as the "destination." (The same computer can be both a source and destination at different times. This is especially clear when one thinks of sending and receiving e-mail.)

Every computer on the Internet has a unique Internet "address" that identifies it from among the millions of computers. The Internet has specialized computers between the source and destination located at network inter-connection points. These computers are known as "routers." The routers understand how to use a computer's address to appropriately point information from one computer to another over the Internet.

In an exchange of information the following occurs:

* The source finds the address of the destination.

* The source contacts the destination and says "hello". 
* The destination responds back with a "hello" of its own.

* The source tells the destination that it has information to send.

* The destination tells the source that it is ready to receive the information.

* The source breaks the information into small pieces called packets and sends each packet on its way to the destination.

* The routers guide each packet to the destination.

* The destination takes the packets and puts them back together to form the information.

* The destination tells the source that it has received the information and asks the source if it has anything more to send.

* If the source says no, the destination will say "good bye" unless it has something to send back. If it does, it will break the information into packets and send them.

* Once both end users are done "talking", they say both say "good bye" .

Clearly our simplified introduction to this section did not explain many steps in this process, such as how a computer discovers the address of another computer or how packets are divided and reassembled. Fortunately, these are specifics that people using the Internet never really need to deal with!

\subsection{Who Runs the Internet?}

No one. The Internet is a cooperative effort among Internet Service Providers (ISPS), software companies, volunteer organizations, and a few facilities that tie the whole thing together. The ISPs and software companies are completely independent and most of them compete with each other. The ISPs provide internet service to people much the same way that they obtain telephone service from a telephone company. ISPs agree to connect their networks to each other and transmit information following an established set of rules (protocols). The software companies agree to manufacture programs (such as email or web browsers) that also follow protocols. There are other organizations that keep things straight. Some assign Internet addresses in much the same manner as telephone numbers are assigned, others keep track of names used by Internet users and groups, and a large volunteer organization called the Internet 
Engineering Task Force (IETF) develops the protocols computers follow to make network communications succeed.

3. Security Considerations

The question "is the Internet secure?" can be a confusing one for people, who will hear many assurances that it is secure and many scary stories saying it is not secure. There are a few basic rules of thumb to remember that will address most concerns.

First, make it a rule never to share account passwords with anyone. Learning a password is the easiest way for someone to break into a system. Most people feel that their files are not that interesting to anyone, but someone may be able to get a foothold from one innocuous account to other places in the same computer system. Many good security practices can be found in the User's Security Handbook [FYI 34, RFC 2504].

Second, understand that there are means for people to track the information a user sends via email, the files one downloads, and the sites visited on the web. The system administrators and network engineers who oversee a sites' computers require access to information that an individual may think is secret. In practice, no responsible system administrator or network engineer will violate a person's privacy out of personal curiosity. However, if someone less legitimate attains illegal access to a system, they also will have access to this information. This situation is not a problem for most people, but it should be understood that things like email sent a year ago or a log of users web pages browsed may still exist in some system's backup archive tape and can be easily resurrected and published widely.

Third, before giving personal information over the Internet, such as filling in a form on a Web page, users should realize that there is no assurance of confidentiality or privacy. It could be compared to faxing such information to a party that you've never dealt with before. While many organizations on the Internet are responsible with information received via the web and email, this cannot always be determined in advance.

4. References

[1] Guttman, E., Leong, G. and G. Malkin, "Users' Security Handbook", FYI 34, RFC 2504, February 1999.

[2] Hambridge, S., "Netiquette Guidelines", FYI 28, RFC 1855, October 1995 . 
5. Authors' Addresses

Raymond Plzak

SAIC

1710 Goodridge Drive

McLean, Virginia 22102

Phone: (703) 821-6535

EMail: plzakresaic.com

Amy Tracy Wells

Internet Scout Project

University of Wisconsin-Madison

Computer Sciences Department

1210 W. Dayton St.

Madison, WI 53706

Phone: (608)263-2611

EMail: awelecs.wisc.edu

Ed Krol

University of Illinois

1120 DCL

1304 Springfield

Urbana IL 61801

Phone (217) 333-7886

EMail: krol@uiuc.edu 


\begin{tabular}{|c|c|}
\hline Glossary of & Terms \\
\hline Emoticon & $\begin{array}{l}\text { Combination of punctuation marks used to provide sense } \\
\text { of the senders tone of voice in an e-mail message }\end{array}$ \\
\hline IETF & $\begin{array}{l}\text { Internet Engineering Task Force [see text for a } \\
\text { description] }\end{array}$ \\
\hline Internet & An interconnected group of networks \\
\hline ISP & Internet Service Provider [see text for a description] \\
\hline Network & A connected group of computers \\
\hline Router & $\begin{array}{l}\text { A specialized computer that connects networks together } \\
\text { and guides information packets to their destination }\end{array}$ \\
\hline Spam & A slang term for junk e-mail \\
\hline URL & Uniform Resource Locator [see text for a description] \\
\hline Web Browser & $\begin{array}{l}\text { A program that provides the capablility to read } \\
\text { information that is located at a world wide web sit }\end{array}$ \\
\hline
\end{tabular}


6. Full Copyright statement

Copyright (C) The Internet Society (1999). All Rights Reserved.

This document and translations of it may be copied and furnished to others, and derivative works that comment on or otherwise explain it or assist in its implementation may be prepared, copied, published and distributed, in whole or in part, without restriction of any kind, provided that the above copyright notice and this paragraph are included on all such copies and derivative works. However, this document itself may not be modified in any way, such as by removing the copyright notice or references to the Internet society or other Internet organizations, except as needed for the purpose of developing Internet standards in which case the procedures for copyrights defined in the Internet Standards process must be followed, or as required to translate it into languages other than English.

The limited permissions granted above are perpetual and will not be revoked by the Internet society or its successors or assigns.

This document and the information contained herein is provided on an "AS IS" basis and THE INTERNET SOCIETY AND THE INTERNET ENGINEERING TASK FORCE DISCLAIMS ALL WARRANTIES, EXPRESS OR IMPLIED, INCLUDING BUT NOT LIMITED TO ANY WARRANTY THAT THE USE OF THE INFORMATION HEREIN WILL NOT INFRINGE ANY RIGHTS OR ANY IMPLIED WARRANTIES OF MERCHANTABILITY OR FITNESS FOR A PARTICULAR PURPOSE.

Acknowledgement

Funding for the RFC Editor function is currently provided by the Internet society. 